\title{
Modeling of Acoustic Emission Signals Generated in On Load Tap Changer
}

\author{
A. Cichoñ*, S. Borucki And D. WotzkA \\ Faculty of Electrical Engineering, Automatic Control and Computer Science, Opole University of Technology \\ Prószkowska 76, 45-758 Opole, Poland
}

\begin{abstract}
The paper deals with mathematical modeling of acoustic emission signals which are generated in on load tap changers. In power engineering area acoustic emission method is commonly used for diagnosis purposes. Authors in their research works apply it for detection of defects occurring in on load tap changers, which are important elements of power transformers. The acoustic emission method is based on measurements performed by use of wideband piezoelectric transducers that are mounted on transformer tank surface. The registered signal is then analyzed in the time, frequency, and time-frequency domains or is evaluated with computer expert systems for diagnosis purposes. Depending on indicators device technical condition can be determined. The contribution given by the authors in this paper is proposal of a mathematical model, which describes envelope of the measured acoustic emission signal.
\end{abstract}

DOI: 10.12693/APhysPolA.125.1396

PACS: 43.40.-r, 43.40.Vn, 43.58.Wc

\section{Introduction}

The scientifically-research works, completed until now in the field of measurement and results analysis in time and time-frequency domains, confirmed the possibility of using the acoustic emission (AE) method for diagnosing of on load tap changers (OLTC) with a structure of separate selector and power switch [1-5]. Due to the possibility of carrying out the measurements during normal on load operation of the transformer, the acoustic method can be applied in diagnostic systems working on-line. These systems require development of a database base, which contains "fingerprints" of individual OLTC defects. Technical implementation of this database may be formed using different IT tools. One method to create such a database is to develop a mathematical model that allows for digital description of the measured AE signals.

This paper presents results of works relating to application of selected regression functions for envelope description of AE signals generated by the power switch.

\section{The object under study and AE signal generated by OLTC}

The process of modeling was applied for AE signals that have been registered for different types of defects simulated under laboratory conditions. They have been characterized in $[1,2,6,7]$. In systems modeling various types of defects the $\mathrm{AE}$ signals were registered and then the envelope of each time course was determined. $\mathrm{AE}$ signals generated by a power switch contain three characteristic structures: S1, S2, S3, which correspond

\footnotetext{
*corresponding author; e-mail: a.cichon@po.opole.pl
}

to the successive acoustic events, wherein the first structure S1, in certain cases, consists of two sub-structures: S1A and S1B. An example of AE signal time course generated by a power switch with new contacts is shown in Fig. 1. The shape of envelope along with the selected structures S1A, S1B, S2, and S3 is shown in Fig. 2.

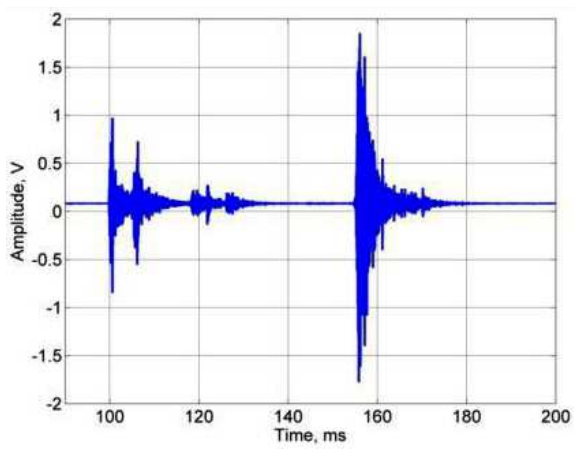

Fig. 1. Time run of the AE signal generated by the OLTC.

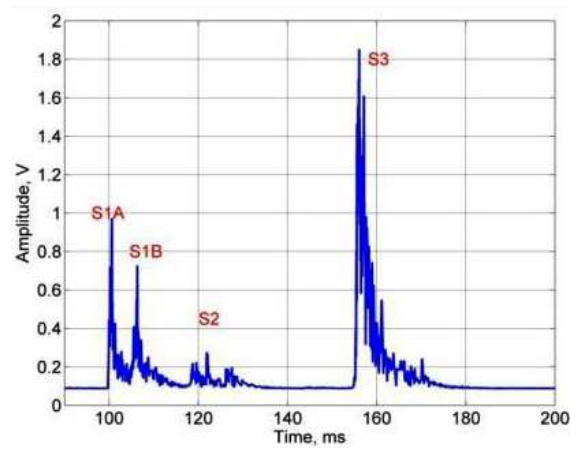

Fig. 2. Time run of the AE signal envelope. The OLTC case with the characteristic structures marked as S1A and S1B, S2 and S3. 


\section{Regression analysis and the mathematical function applied}

Envelopes of AE signals generated by the switch may be approximated with a function $y(t)$. In the process of parameter estimation of function $y(t)$ a non-linear regression function model was used, which is described by Eq. (1):

$$
\hat{Y}=f(X, P)+\xi
$$

where $\hat{Y}$ - theoretical value of the regression function $y(t)=f(X, P), X$ - set of dependent and independent variables, $P$ - set of parameters, $\xi-$ random coefficient [8].

The parameters of the regression function were estimated by means of least squares method. The estimates were then used in optimization process, in which the Nelder-Mead-Simplex method was applied [9]. The estimation process was performed complying the minimal value of the residual norm $\delta$, which is described by Eq. (2):

$$
\delta=\|\hat{\boldsymbol{y}}-\boldsymbol{y}\|=\sqrt{\sum_{i=1}^{n}\left(\hat{y}_{i}-y_{i}\right)},
$$

where $\delta$ - value of the residual norm $\|\hat{\boldsymbol{y}}-\boldsymbol{y}\|, \hat{y}_{i}-i$-th estimate of the regression function, $y_{i}$ - empirical data, $i=1 \ldots n, n=50000$ - number of samples.

In order to describe the signal envelope with a mathematical model, ten different nonlinear regression functions (shortly called models) were considered:

1. Levy type function (LEV).

2. Birnbaum-Sanders type function $(B-S)$.

3. Sigmoid type function (SIG)

4. Nakagami type function (NAK)

5. Generalized extreme value type function (GEV).

The parameterization and optimization processes using proposed regression functions was applied for envelopes of the individual structures: S1, S2, S3, occurring in the analyzed signals. As estimates for regression functions, by which one can describe the envelopes of AE signals generated by power switch, we proposed five mathematical models with one dependent and one independent variable. The dependent variable is the actual value of the registered AE signal envelope, while the independent variable is time. The adopted models are characterized by similar course to the assigned functions.

\subsection{Levy type function $L E V$}

The regression function of type Levi is expressed by Eq. (3). Figure 3 shows the time course of envelope of the S1A structure of the AE signal generated by OLTC with a modeled defect, and the calculated regression curve LEV:

$$
f(x)=A \sqrt{\frac{\gamma}{2 \pi}} \frac{1}{(x-\mu)^{3} / 2} \exp \left(-\frac{\gamma}{2(x-\mu)}\right),
$$

where $f(x)$ - estimate of the regression function LEV, $A \in R, \mu \in R, \gamma>0, x>\mu \sigma$ - function parameters estimates, $x$ - independent variable, time.

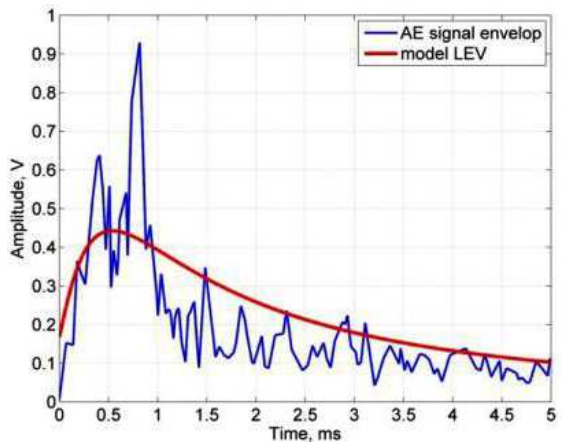

Fig. 3. Time run of the AE signal envelope and the LEV model applied for the S1A structure.

\subsection{Birnbaum-Sanders type function $B-V$}

The regression function of type Birnbaum-Sanders is expressed by Eq. (4). Figure 4 shows the time course of envelope of the S1A structure of the AE signal generated by OLTC with a modeled defect, and the calculated regression curve $\mathrm{B}-\mathrm{S}$ :

$$
\begin{aligned}
f(x) & =\frac{A}{\sqrt{2 \pi}} \exp \left(-\frac{(\sqrt{x / \beta}-\sqrt{\beta / x})^{2}}{2 \gamma^{2}}\right) \\
& \times \frac{\sqrt{x / \beta}+\sqrt{\beta / x}}{2 \gamma x},
\end{aligned}
$$

where $f(x)$ - estimate of the regression function $\mathrm{B}-\mathrm{S}$, $A \in R, x \in R, \gamma>0, \beta>0$ - function parameters estimates, $x$ - independent variable, time.

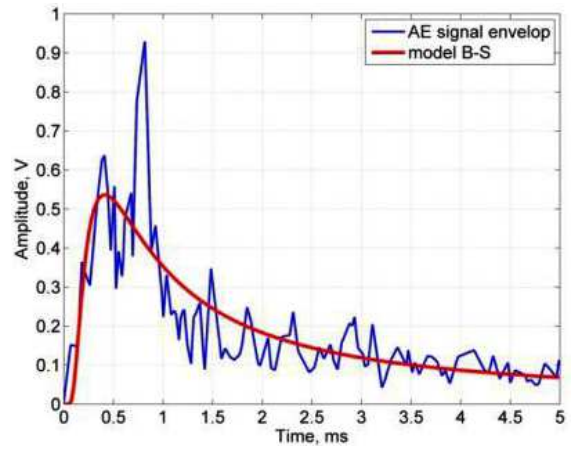

Fig. 4. Time run of the AE signal envelope and the B-S model applied for the S1A structure.

\subsection{Sigmoid type function $S I G$}

The regression function of type sigmoid is expressed by Eq. (5). Figure 5 shows the time course of envelope of the S1A structure of the AE signal generated by OLTC with a modeled defect, and the calculated regression curve SIG: 


$$
\begin{aligned}
& f(x)=A\left[a_{1} \exp \left(-\left(\frac{\left(x-\mu_{1}\right)}{c_{1}}\right)^{2}\right)\right. \\
& +a_{2} \exp \left(-\left(\frac{\left(x-\mu_{2}\right)}{c_{2}}\right)^{2}\right) \\
& \left.+a_{3} \exp \left(-\left(\frac{\left(x-\mu_{3}\right)}{c_{3}}\right)^{2}\right)\right] \\
& \times \frac{1}{1+k \exp \left(-b\left(x-\mu_{4}\right)\right)},
\end{aligned}
$$

where $f(x)$ - estimate of the regression function SIG, $\left(A, a_{i}, b_{i}, c_{i}\right) \in R$, for $i=\{1 \ldots 3\}, x \in R, k>0, b$, $\left(\mu_{i}\right) \in R$, for $i=\{1 \ldots 4\}$ - function parameters estimates, $x$ - independent variable, time.

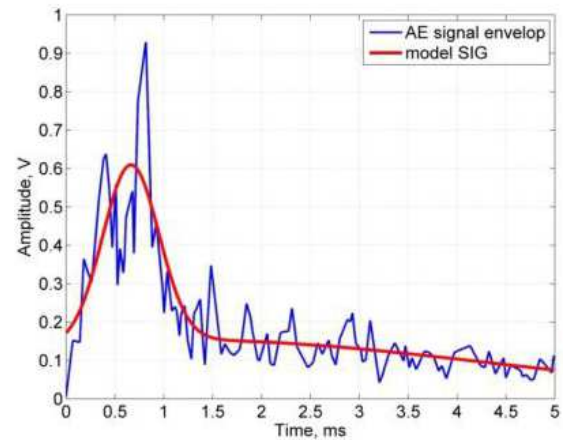

Fig. 5. Time run of the AE signal envelope and the SIG model applied for the S1A structure.

\subsection{Nakagami type function $N A K$}

The regression function of type Nakagami is expressed by Eq. (6). Figure 6 shows the time course of envelope of the S1A structure of the AE signal generated by OLTC with a modeled defect, and the calculated regression curve NAK:

$$
f(x)=A\left(\frac{\mu}{\omega}\right)^{\mu} \frac{1}{\Gamma(\mu)} x^{2 \mu-1} \exp \left(-\frac{\mu}{\omega} x^{2}\right),
$$

where $f(x)$ - estimate of the regression function NAK, $A \in R, x \in R, x>0, \omega>0, \omega \in R, \mu \in R, \mu \geq 0.5$

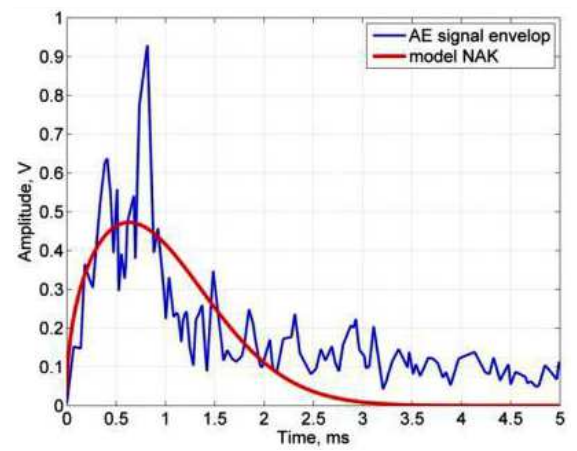

Fig. 6. Time run of the AE signal envelope and the NAK model applied for the S1A structure.
- function parameters estimates, $x$ — independent variable, time.

\subsection{Generalized extreme value type function $G E V$}

The regression function of type generalized extreme value is expressed by Eq. (7). Figure 7 shows the time course of envelope of the S1A structure of the AE signal generated by OLTC with a modeled defect, and the calculated regression curve GEV:

$$
\begin{aligned}
& f(x)=\frac{A}{\sigma} \exp \left(-\left(1+k \frac{x-\mu}{\sigma}\right)^{-\frac{1}{k}}\right) \\
& \times\left(1+k \frac{x-\mu}{\sigma}\right)^{-1-\frac{1}{k}},
\end{aligned}
$$

where $f(x)$ - estimate of the regression function GEV, $A \in R, x \in R, x \geq 0, \sigma>0, \mu \in R, k \in R$ - function parameters estimates, $x-$ independent variable, time.

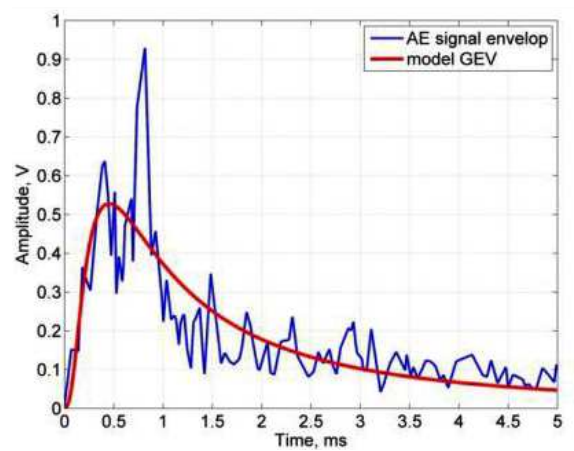

Fig. 7. Time run of the AE signal envelope and the GEV model applied for the S1A structure.

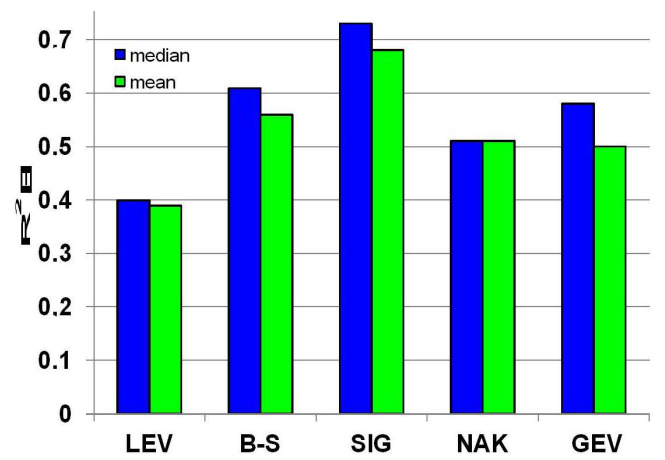

Fig. 8. Arithmetic mean and median values of the correlation coefficient $R^{2}$, determined for five models.

As a fitting measure of estimated regression function parameters for each structure occurring in the modeled AE signal envelopes, the determination coefficient value $R^{2}$ was considered. For the calculated values of $R^{2}$ the arithmetic mean and median were estimated and are shown for comparison in form of column chart in Fig. 8. 


\section{Summing-up}

Based on a comparative analysis of determination coefficient values $R^{2}$, two of the five mathematical models used were highlighted: B-S and SIG. They are characterized by the greatest degree of fitting to the empirical parameters, which was confirmed by the calculated arithmetic means of determination coefficient $R^{2}$, which are as follows: for the $\mathrm{B}-\mathrm{S}$ model mean $=0.56$, for the $\mathrm{SIG}$ model mean $=0.68$. It has been shown that the highest value of determination coefficient was achieved for the SIG model. On the basis of these results for further analyses, associated with development of a mathematical equation for the entire envelope of $\mathrm{AE}$ signal generated by the power switch, it is planned to use the SIG model.

\section{References}

[1] A. Cichoń, Studies and Monographs, Wydawnictwo Politechniki Opolskiej, Vol. 259, Opole 2011 (in Polish).
[2] A. Cichoń, Studies and Monographs, Wydawnictwo Politechniki Opolskiej, Vol. 352, Opole 2013 (in Polish).

[3] A. Cichoń, P. Berger, S. Borucki, Measurments Automatics Control 2, 152 (2013) (in Polish).

[4] Shen Dazhong, A. Kramer, D. Dohnal, in: Int. Conf. on Power Systems Technology, POWERCON 2006, Chongqing (China), 2006, p. 1.

[5] P. Kang, Ph.D. Thesis, Queensland Univ. Technol., Brisbane, Australia 2000.

[6] A. Cichoń, Electric Rev. 4a, 22 (2012) (in Polish).

[7] A. Cichoń, S. Borucki, D. Wotzka, M. Szmajda, Acta Phys. Pol. A 122, 804 (2012).

[8] J.S. Bendat, A.G. Piersol, Engineering Application of Correlation and Spectral Analysis, Wiley, New York 1993.

[9] J.C. Lagarias, J.A. Reeds, M.H. Wright, P.E. Wright, SIAM J. Optimiz. 9, 112 (1998). 